\title{
Simplified Adaptive Robust Motion Control with Varying Boundary Discontinuous Projection of Hydraulic Actuator
}

\author{
Cungui Yu and Xianwei Qi \\ School of Mechanical Engineering, Nanjing University of Science and Technology, Nanjing 210094, China \\ Correspondence should be addressed to Cungui Yu; yu-cungui@njust.edu.cn
}

Received 26 June 2014; Accepted 8 July 2014; Published 24 July 2014

Academic Editor: Weichao Sun

Copyright ( 2014 C. Yu and X. Qi. This is an open access article distributed under the Creative Commons Attribution License, which permits unrestricted use, distribution, and reproduction in any medium, provided the original work is properly cited.

\begin{abstract}
This paper deals with the high performance adaptive robust motion control of electrohydraulic servo system driven by dual vane hydraulic rotary actuator. The recently developed adaptive robust control theory is used to handle the nonlinearities and modelling uncertainties in hydraulic systems. Aside from the difficulty of handling parametric variations, the traditional adaptive robust controller (ARC) is also a little complicated in practice. To address these challenging issues, a simplified adaptive robust control with varying boundary discontinuous projection is developed to enhance the robustness of the closed-loop system, based on the features of hydraulic rotary actuator. Compared with previous ARC controller, the resulting controller has a simple algorithm for more suitable implementation and can handle parametric variations via nonlinear robust design. The controller theoretically achieves a guaranteed transient performance and final tracking accuracy in the presence of both parametric uncertainties and uncertain nonlinearities. Extensive simulation results are obtained for a hydraulic rotary actuator to verify the high performance nature of proposed control strategy.
\end{abstract}

\section{Introduction}

Hydraulic systems have been used in industry in a wide number of applications by virtue of their small size-to-power ratios and the ability to apply very large forces and torques, for example electrohydraulic positioning systems [1-4], vehicle active suspensions [5-7], hydraulic motion simulators [8], hydraulic force systems [9-15], and so on. As a branch of hydraulic systems, hydraulic rotary actuators are usually applied in direct rotary drive applications [16-18]. However, nonlinear behaviours [19], stability, and parameter variations during operation $[20,21]$ continue to complicate the development of high performance closed-loop controllers, and it is still a difficult task to develop an advanced controller to address all parametric uncertainties and uncertain nonlinearities [2] meanwhile have a simple structure for feasible implementation. This leads to the urgent need for advanced hydraulic control technologies.

In the past, lots of research works in control of hydraulic systems have used linear control theory and feedback linearization techniques [22]. Although these linearization control methods work well for some systems, for a highly nonlinear and uncertain system, they may not achieve acceptable control performance in all conditions. To improve the tracking performance for nonlinear and uncertain systems, various robust and/or adaptive controllers have been applied to uncertain nonlinear systems [23-25], including hydraulic servo systems. These nonlinear control schemes can achieve better performance than conventional linear controllers.

During the past two decades, a mathematically rigorous nonlinear adaptive robust control (ARC) approach has been proposed for high performance robust control of uncertain nonlinear systems in the presence of both parametric uncertainties and uncertain nonlinearities [26-28]. The developed ARC theory bridges the gap between two of the main control research areas [29]_adaptive control (AC) [30, 31] and deterministic robust control (DRC) [32]. By integrating the fundamentally different working mechanisms of the two approaches, the developed ARC theory is able to preserve the theoretical performance results of both design approaches while overcoming their well-known practical performance limitations [29]. Up to now, there are many successful applications to physical systems [33-36] by applying ARC 

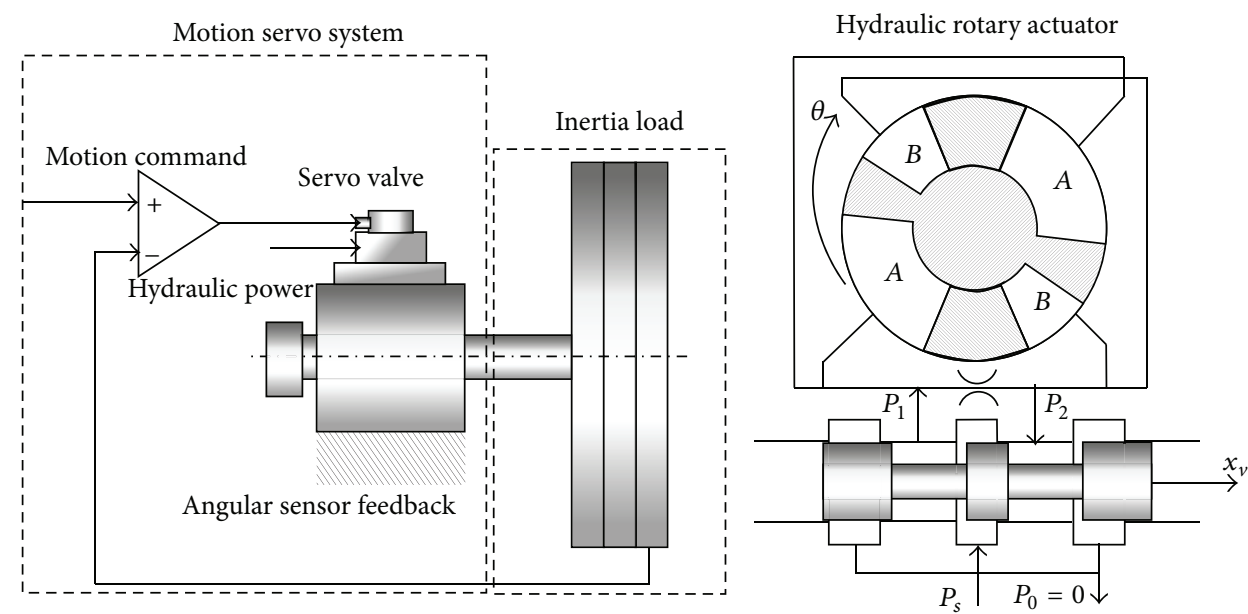

FIGURE 1: Hydraulic motion servo system and rotary actuator.

control theory with some modifications suitable for actual systems.

For the hydraulic system, its relative degree is normally larger than one and also has nonsmooth nonlinearities, such as frictions [18], complicated internal leakage characteristics [1], and flow nonlinearities of directional change of valve opening [19]; the previous ARC approach has to carry out the back-stepping design via Lyapunov functions with discontinuous projection method [2]. However, as known in [37], it is difficult for direct adaptive robust control to ensure the parameter estimation converging or even approaching to their true values fast enough as observed in actual applications [2, 38]. Although integrated direct/indirect ARC method not only guarantees accurate estimation of physical parameters but also introduces dynamic compensation to achieve better transient and steady-state performance [39], it is very difficult to be implemented for hydraulic systems together with backstepping designs.

In this paper, a simplified adaptive robust control with varying boundary discontinuous projection strategy is proposed. In contrast to the previous ARC controllers, in order to improve the operability, the simplified ARC approach reduces the relative degree of hydraulic servo system to one by utilizing the feature that hydraulic rotary actuator typically has larger leakage characteristics, which can help to avoid carrying out the complicated backstepping design. Moreover, the varying boundary discontinuous projection will be used to handle the estimation process of varying parameters via differential pressure signal. As a result, the resulting controller becomes simpler and the parameter adaptation process is more comprehensive in the presence of varying parameters.

To test the proposed simplified ARC strategy with varying boundary discontinuous projection, extensive comparative simulation results have been obtained for the motion control of a hydraulic rotary actuator. These results verify the highperformance nature of the proposed approach as same as traditional ARC method, but have more simple structure and flexible parametric adaptation.

The remainder of this paper is organized as follows. Problem formulation and system models are given in Section 2.
Controller design and its theoretical results are present in Section 3. Simulation results are obtained in Section 4. And conclusions can be found in Section 5.

\section{Problem Formulation and Dynamic Models}

The system under consideration is depicted in Figure 1. The goal is to have the inertia load to track any smooth motion trajectory as closely as possible. The dynamics of the inertia load can be described by

$$
J_{m} \ddot{\theta}=P_{L} D_{m}-B_{m} \dot{\theta}+T_{\mathrm{ed}}+\tilde{f}(t, \theta, \dot{\theta})
$$

where $\theta$ and $J_{m}$ represent the rotary angle and the inertia of the load, respectively, $P_{L}=P_{1}-P_{2}$ is the load pressure of the hydraulic rotary actuator, $D_{m}$ is the volumetric displacement of the hydraulic rotary actuator, $B_{m}$ represents the combined coefficient of the modelled damping and viscous friction torques on the load and the rotary actuator shaft, $T_{\text {ed }}$ represents the external disturbance, and $\widetilde{f}(t, \theta, \dot{\theta})$ represents other disturbances like the unmodeled friction torques. Neglecting the internal leakage in the servo valve, the pressure dynamics can be written as follows [19]:

$$
\frac{V_{m}}{4 \beta_{e}} \dot{P}_{L}=-D_{m} \dot{\theta}-C_{\mathrm{tm}} P_{L}+Q_{L},
$$

where $V_{m}$ is the total volume of the hydraulic rotary actuator and the hoses between the actuator and the servo valve, $\beta_{e}$ is the effective bulk modulus, $C_{\mathrm{tm}}$ is the coefficient of the total internal leakage of the actuator due to pressure, and $Q_{L}$ is the load flow. $Q_{L}$ is related to the spool valve displacement of the servo valve, $x_{v}$, by [19]

$$
Q_{L}=C_{d} w x_{v} \sqrt{\frac{P_{s}-\operatorname{sgn}\left(x_{v}\right) P_{L}}{\rho}},
$$


which can be rewritten as follows:

$$
\begin{gathered}
Q_{L}=K_{Q} x_{v}, \\
K_{Q}=C_{d} w \sqrt{\frac{g\left(P_{L}, x_{v}\right)}{\rho},} \\
g\left(P_{L}, x_{v}\right) \triangleq P_{s}-\operatorname{sgn}\left(x_{v}\right) P_{L},
\end{gathered}
$$

where $K_{Q}$ is the varying flow rate of the servo valve, $C_{d}$ is the discharge coefficient, $w$ is the spool valve area gradient, $\rho$ is the density of oil, $P_{s}$ is the supply pressure of the fluid, and $\operatorname{sgn}(\cdot)$ is the sign function. The spool valve displacement $x_{v}$ is related to the current input $i$ by a first order system given by [2]

$$
\tau_{v} \dot{x}_{v}=-x_{v}+K_{v} i
$$

where $\tau_{v}$ and $K_{v}$ are the time constant and electrical gain of the servo valve, respectively. Define the state variable $x=\left[x_{1}, x_{2}, x_{3}, x_{4}\right]^{T} \triangleq\left[\theta, \dot{\theta}, P_{L}, x_{v}\right]$, then the entire system equations (1), (2), (4), and (5) can be rewritten in state space form as follows:

$$
\begin{aligned}
\dot{x}_{1} & =x_{2}, \\
\dot{x}_{2} & =\frac{1}{J_{m}}\left[D_{m} x_{3}-B_{m} x_{2}+T_{\mathrm{ed}}+d\left(t, x_{1}, x_{2}\right)\right], \\
d\left(t, x_{1}, x_{2}\right) & =\frac{1}{J_{m}} \tilde{f}\left(t, x_{1}, x_{2}\right), \\
\dot{x}_{3} & =\frac{4 \beta_{e}}{V_{m}}\left(-D_{m} x_{2}-K_{\mathrm{tm}} x_{3}+K_{\mathrm{Q}} x_{4}\right), \\
\dot{x}_{4} & =-\frac{1}{\tau_{v}} x_{4}+\frac{K_{v} K_{v i}}{\tau_{v}} u,
\end{aligned}
$$

where $u=i / K_{v i}$ is the control input voltage and $K_{v i}$ is the coefficient of the conversion gain from voltage to electric current.

Given the desired motion trajectory $y_{d}=x_{1 d}(t)$, the objective is to synthesize a control input $u$ such that the output $y=x_{1}$ tracks $x_{1 d}(t)$ as closely as possible in spite of various modelling uncertainties and external disturbances.

\section{Simplified Adaptive Robust Control and Varying Boundary Discontinuous Projection of Electrohydraulic Servo Systems}

3.1. Simplified Design Model and Issues to Be Addressed. From [2], it can be seen that the resulting controller given in [2] is very complicated since the relative degree of the employed model is more than one, that is, to say, the backstepping design via Lyapunov functions has to be employed for controller design. The application of the nonsmooth equation of the load flow related to the displacement of the spool of servo valve makes the final resulting controller too complicated to implement. Checking the experimental results in $[1,2]$ carefully, some simplifications can be made for implementation, as done in what follows.
To begin the controller design, some practical and reasonable assumptions and simplifications have to be made. For simplicity, the dynamic (5) can be replaced by a proportional element since the high bandwidth servo valve is usually employed in the high performance hydraulic servo system. Then dynamic (5) turns into

$$
x_{v}=K_{v} i .
$$

Also, combined with load flow equation (4), the dynamic equation (2) can be rewritten as follows:

$$
P_{L}=\frac{1}{\left(V_{m} / 4 \beta_{e}\right) s+C_{\mathrm{sl}}}\left(K_{\mathrm{Q}} x_{v}-D_{m} \theta s\right),
$$

where $s$ is the differential operator. Typically, the dual vane hydraulic rotary actuator usually has a larger internal leakage between its two chambers than hydraulic cylinder because of its inherent irregular sealing. And then the following condition is normally satisfied:

$$
\frac{V_{m}}{4 \beta_{e} C_{\mathrm{sl}}}<\sigma
$$

where $\sigma$ is a very small constant, such as 0.01 . Then the dynamic response in (8) is fast enough to be ignored. That means (2) can be simplified as follows:

$$
P_{L}=\frac{1}{C_{\mathrm{sl}}}\left(K_{\mathrm{Q}} x_{v}-D_{m} \dot{\theta}\right) .
$$

Regarding the discontinuous nonlinearity due to the directional change of valve opening, $g\left(P_{L}, x_{v}\right)$ can be calculated by using the load pressure signal $P_{L}$. Combining (7), the state space equation (6) can be simplified as follows:

$$
\begin{aligned}
& \dot{x}_{1}=x_{2}, \\
& \dot{x}_{2}=\frac{1}{J_{m}} \frac{D_{m}}{C_{s l}}\left(K_{u} u-D_{m} x_{2}-\frac{C_{\text {sl }}}{D_{m}} B_{m} x_{2}+\frac{C_{\text {sl }}}{D_{m}} T_{\text {ed }}+\frac{C_{\text {sl }}}{D_{m}} d\right), \\
& K_{u}=K_{\text {to }} g\left(P_{L}, u\right), \quad K_{\text {to }} \triangleq C_{d} w \sqrt{\frac{1}{\rho}} K_{v} K_{v i},
\end{aligned}
$$

where $K_{u}$ is the voltage-flow gain of the servo valve and can be calculated by sensing the load pressure $P_{L}$. At this stage, it is easy to see that the simplified system state space equation (11) is of second order and only has matched uncertainties, that is, the relative degree is one. This simplification avoids the backstepping design.

As in [2], to minimize the numerical error and facilitate the gain-tuning process, constant scaling factors are introduced to the system as follows:

$$
\begin{aligned}
\bar{J}_{m} & =S_{c} J_{m}=\frac{C_{\mathrm{sl}}}{D_{m}} J_{m}, \quad \bar{B}_{m}=D_{m}+S_{c} B_{m}=D_{m}+\frac{C_{\mathrm{sl}}}{D_{m}} B_{m}, \\
\bar{T}_{\text {ed }} & =S_{c} T_{\text {ed }}=\frac{C_{\text {sl }}}{D_{m}} T_{\text {ed }}, \quad \widetilde{d}=S_{c} d=\frac{C_{\text {sl }}}{D_{m}} d, \\
u & =\frac{\bar{u}}{K_{u}} .
\end{aligned}
$$


In the above scaling expressions, $S_{c 1}=C_{s l} / D_{m}$ is a constant scaling factor.

In general, the system (11) is also subjected to parametric uncertainties due to the variations of $J_{m}, D_{m}, C_{\mathrm{sl}}, B_{m}$, and $K_{\mathrm{to}}$. In reality, some parameters can usually be known or calculated, like $D_{m}, C_{\mathrm{sl}}$, and $K_{\text {to }}$. So in this paper, we only consider the parametric uncertainties of load parameters like $J_{m}$ and $B_{m}$, which aredifficult to be calculated or known, and the external disturbance $T_{\text {ed }}$. Other parametric uncertainties can be dealt with in the same way if necessary. In order to use parameter adaptation to reduce parametric uncertainties and improve tracking performance, define the unknown parameter set $\psi=\left[\psi_{1}, \psi_{2}, \psi_{3}\right]^{T}$ as follows:

$$
\psi_{1}=\bar{J}_{m}, \quad \psi_{2}=\bar{B}_{m}, \quad \psi_{3}=\bar{T}_{\mathrm{ed}} .
$$
follows:

The state space equation (11) can thus be rewritten as

$$
\begin{aligned}
& \dot{x}_{1}=x_{2}, \\
& \dot{x}_{2}=\frac{1}{\psi_{1}}\left[\bar{u}-\psi_{2} x_{2}+\psi_{3}+\tilde{d}\left(t, x_{1}, x_{2}\right)\right] .
\end{aligned}
$$

For most applications, the extent of the parametric uncertainties and uncertain nonlinearities is known. Thus the following practical assumption is made.

Assumption 1. Parametric uncertainties and uncertain nonlinearities satisfy

$$
\begin{gathered}
\psi \in \Omega_{\psi} \triangleq\left\{\psi: \psi_{\min }<\psi<\psi_{\max }\right\}, \\
\left|\widetilde{d}\left(t, x_{1}, x_{2}\right)\right| \leq \delta_{d}\left(t, x_{1}, x_{2}\right),
\end{gathered}
$$

where $\psi_{\min }=\left[\psi_{1 \text { min }}, \psi_{2 \min }, \psi_{3 \min }\right]^{T}, \psi_{\max }=\left[\psi_{1 \text { max }}, \psi_{2 \max }\right.$, $\left.\psi_{3 \max }\right]^{T}$, and $\delta_{d}\left(t, x_{1}, x_{2}\right)$ are known.

In (16), $\bullet$ i represents the $i$ th component of the vector $\bullet$ and the operation $<$ for two vectors is performed in terms of the corresponding elements of the vectors.

In the following, a varying boundary discontinuous projection based ARC controller will be presented to achieve high performance of hydraulic servo system. To this end, the following notations are introduced.

3.2. Notations and Varying Boundary Discontinuous Projection Mapping. Let $\widehat{\psi}$ denote the estimate of $\psi$ and let $\widetilde{\psi}$ denote the estimation error (i.e., $\widetilde{\psi}=\widehat{\psi}-\psi$ ). Viewing (15), a varying boundary discontinuous projection can be defined as follows:

$$
\operatorname{Proj}_{\widehat{\psi}_{i}}\left(\bullet_{i}\right)= \begin{cases}0 & \text { if } \widehat{\psi}_{i}=\phi_{i \max }(t, \psi, x), \bullet_{i}>0 \\ 0 & \text { if } \widehat{\psi}_{i}=\phi_{i \min }(t, \psi, x), \bullet_{i}<0 \\ \bullet_{i} & \text { otherwise, }\end{cases}
$$

where $i=1, \ldots, 3$ and $\phi_{i \text { max }}(t, \psi, x)$ and $\phi_{i \text { min }}(t, \psi, x)$ are the maximal and minimum varying boundary of parameter $\psi$, respectively, that is,

$$
\phi_{i \max }(t, \psi, x)<\psi_{\max }, \quad \psi_{\min }<\phi_{i \min }(t, \psi, x) \quad \forall t, \psi, x,
$$

and an example for the varying boundaries will be given in Section 4.

By using an adaptation law given by

$$
\dot{\widehat{\psi}}_{i}=\operatorname{Proj}_{\widehat{\psi}_{i}}(\Gamma \tau) \text {, }
$$

where $\operatorname{Proj}_{\widehat{\psi}_{i}}(\bullet)=\left[\operatorname{Proj}_{\widehat{\psi}_{i}}\left(\bullet_{1}\right), \ldots, \operatorname{Proj}_{\widehat{\psi}_{i}}\left(\bullet_{3}\right)\right]^{T}, \Gamma>0$ is a diagonal matrix and $\tau$ is an adaptation function to be synthesized later. As similar to that shown in $[28,40]$ for any adaption function $\tau$, the projection mapping used in (18) guarantees

$$
\begin{aligned}
& \widehat{\psi} \in \bar{\Omega}_{\psi} \triangleq\left\{\widehat{\psi}: \psi_{\min }<\widehat{\psi}<\psi_{\max }\right\}, \\
& \widehat{\psi} \in \bar{\Omega}_{\psi} \triangleq\left\{\widehat{\psi}: \phi_{\min }(t, \psi, x)<\widehat{\psi}<\phi_{\max }(t, \psi, x)\right\}, \\
& \widetilde{\psi}^{T}\left(\Gamma^{-1} \operatorname{Proj}_{\widehat{\psi}}(\Gamma \tau)-\tau\right) \leq 0, \forall \tau .
\end{aligned}
$$

Properties (P1) and (P2) imply that the parameter estimations are always within the known bounded set $\bar{\Omega}_{\psi}$ and the given varying boundary, that is, $\phi_{\min }(t, \psi, x)$ and $\phi_{\max }(t, \psi, x)$. Property (P3) enables one to know that the use of projection modification to the discontinuous adaptation law holds the perfect learning capability of the traditional one. Define a function $E(\vec{A}, \vec{B})=\|\vec{A}-\vec{B}\|$ and make $E T_{\max }=\max \left\{E\left(\psi, \psi_{\max }\right), E\left(\psi, \psi_{\min }\right)\right\}, E V_{\max }=\max \{E(\psi$, $\left.\left.\phi_{\max }(t, \psi, x)\right), E\left(\psi, \phi_{\min }(t, \psi, x)\right)\right\}$. Combining Assumption 1, inequality (17), and properties (P1) and (P2) in (19), it is obvious that

$$
\text { (P4) } \quad\|\widetilde{\psi}\| \leq E V_{\max } \leq E T_{\max }, \quad \forall t, \psi, x .
$$

Property (P4) shows that a better estimation performance can be given by using the varying boundary discontinuous projection, compared to the traditional discontinuous projection so long as the given varying boundary is reasonable.

3.3. Controller Design. Noting that the first equation of (14) does not have any uncertainties, one step design can thus be constructed for the first two equations of (14) directly. Define a switching function like quantities as follows:

$$
p=\dot{e}+k_{1} e=\dot{y}-\dot{y}_{\mathrm{eq}}, \quad \dot{y}_{\mathrm{eq}}=\dot{y}_{d}-k_{1} e,
$$

where $e=x_{1}-x_{1 d}(t)$ is the output tracking error and $k_{1}$ is any positive feedback gain. Since $G_{s}(s)=e(s) / p(s)=1 /\left(s+k_{1}\right)$ is a stable transfer function, making $e$ small or converging to zero is equivalent to making $p$ small or converging to zero. So the rest of the design is to make $p$ as small as possible with a guaranteed transient performance [2]. Differentiating (21) and noting (14), we have

$$
\bar{J}_{m} \dot{p}=\bar{J}_{m} \ddot{y}-\bar{J}_{m} \ddot{y}_{\mathrm{eq}}=\bar{u}-\bar{B}_{m} \dot{y}+\bar{T}_{\mathrm{ed}}+\widetilde{d}-\bar{J}_{m} \ddot{y}_{\mathrm{eq}} \cdot
$$

With the projection type adaptive law (16), a direct adaptive robust controller can be synthesized for the system (14) to achieve a guaranteed transient and final tracking accuracy. Furthermore, to reduce the effect of measurement noise, 
desired compensation ARC (DCARC) [27] is used in this paper. For the stage to track $y_{d}(t)$ perfectly, it is necessary that the initial conditions are matched, that is,

$$
y_{d}(0)=x_{1}(0), \quad \dot{y}_{d}(0)=\dot{x}_{2}(0),
$$

and the resulting DCARC control law has the following form:

$$
\begin{aligned}
& \bar{u}=\bar{u}_{a}+\bar{u}_{s}, \\
& \bar{u}_{a}=-\varphi_{d}^{T} \widehat{\psi},
\end{aligned}
$$

where $\bar{u}_{a}$ is the adjustable model compensation needed for achieving perfect tracking through online parameter adaptation given by (18), $\varphi_{d}^{T}=\left[-\ddot{y}_{d},-\dot{y}_{d}, 1\right]$ is the regressor that depends on the reference trajectory $y_{d}(t)$ only and thus is free of measurement noise, and $\bar{u}_{s}$ is a robust control law to compensate for some loss of system information having the form of

$$
\begin{gathered}
\bar{u}_{s}=\bar{u}_{s 1}+\bar{u}_{s 2}, \\
\bar{u}_{s 1}=-k_{s 1} p,
\end{gathered}
$$

where $k_{s 1}$ is a nonlinear gain large enough such that the matrix $A_{1}$ defined below is positive definite

$$
A_{1}=\left(\begin{array}{cc}
k_{s 1}-k_{2}-\psi_{1} k_{1}+\psi_{2} & -\frac{1}{2} k_{1} \psi_{2} \\
-\frac{1}{2} k_{1} \psi_{2} & \frac{1}{2} \psi_{1} k_{1}^{3}
\end{array}\right),
$$

where $k_{2}$ is any positive feedback gain and $\bar{u}_{s 2}$ is a robust control function designed as follows. Substituting (24) and (25) into (22)

$$
\bar{J}_{m} \dot{p}=-k_{s 1} p+\left(\bar{J}_{m} k_{1}-\bar{B}_{m}\right) \dot{e}+\bar{u}_{s 2}-\varphi_{d}^{T} \widetilde{\psi}+\widetilde{d} .
$$

The robust control function $\bar{u}_{s 2}$ is now chosen to satisfy the following conditions:

$$
\begin{aligned}
& \text { Condition (i) } \quad p\left[\bar{u}_{s 2}-\varphi_{d}^{T} \widetilde{\psi}+\widetilde{d}\right]<\varepsilon, \\
& \text { Condition (ii) } \quad p \bar{u}_{s 2} \leq 0,
\end{aligned}
$$

where $\varepsilon$ is a design parameter which can be arbitrarily small. Essentially, Condition (i) of (28) shows that $\bar{u}_{s 2}$ is synthesized to dominate the model uncertainties coming from both parametric uncertainties $\widetilde{\psi}$ and uncertain nonlinearities $\widetilde{d}$, and Condition (ii) is to make sure that $\bar{u}_{s 2}$ is dissipating in nature so that it does not interfere with the functionality of the adaptive control part $\bar{u}_{a}$. How to choose $\bar{u}_{s 2}$ to satisfy constraints like (28) can be found in $[26,40]$.

Remark 2. One example of a smooth $\bar{u}_{s 2}$ satisfying (28) can be found in the following way. Let $h$ be any smooth function satisfying

$$
h \geq\left\|\psi_{M}\right\|^{2}\left\|\varphi_{d}\right\|^{2}+\delta_{d}^{2},
$$

where $\psi_{M}=\psi_{\max }-\psi_{\min }$, then, $\bar{u}_{s 2}$ can be chosen as follows:

$$
\bar{u}_{s 2}=-\frac{h}{2 \varepsilon} p .
$$

It can be known that (28) is satisfied [26].
3.4. Main Results. With the control law $\bar{u}$, the following theoretical performance holds.

Theorem 3. With the projection type adaptation law (18) and adaptation function of $\tau=\varphi_{d} p$, the DCARC law (24) guarantees the following.

(A) In general, all signals are bounded. Furthermore, the positive definite function $V_{s}$ defined by

$$
V_{s}=\frac{1}{2} \psi_{1} p^{2}+\frac{1}{2} \psi_{1} k_{1}^{2} e^{2},
$$

is bounded above by

$$
V_{s} \leq \exp (-\lambda t) V_{s}(0)+\frac{\varepsilon}{\lambda}[1-\exp (-\lambda t)],
$$

where $\lambda=\min \left\{2 k_{2} / \psi_{1 \max }, k_{1}\right\}$.

(B) If after a finite time $t_{0}, \widetilde{d}=0$, that is, in the presence of parametric uncertainties only (i.e., $\widetilde{d}=0, \forall t \geq t_{0}$ ), and the disturbance $T_{e}$ is constant, then, in addition to results in (A), asymptotic output tracking (or zero final tracking error) is also achieved, that is, $e \rightarrow 0$ and $p \rightarrow 0$ ast $\rightarrow \infty$.

Proof of Theorem 3. Based on (27), the time derivative of $V_{s}$ is

$$
\dot{V}_{s}=p\left\{\bar{u}_{s}-\varphi_{d}^{T} \widetilde{\psi}+\widetilde{d}+\left(\psi_{1} k_{1}-\psi_{2}\right) \dot{e}\right\}+\psi_{1} k_{1}^{2} e \dot{e} .
$$

Applying (25) and noting that $\dot{e}=p-k_{1} e$, we have

$$
\begin{aligned}
\dot{V}_{s} \leq & p\left\{\bar{u}_{s 2}-\varphi_{d}^{T} \widetilde{\psi}+\widetilde{d}-k_{s 1} p+\left(\psi_{1} k_{1}-\psi_{2}\right) \dot{e}\right\}+\psi_{1} k_{1}^{2} e \dot{e} \\
= & p\left\{\bar{u}_{s 2}-\varphi_{d}^{T} \widetilde{\psi}+\widetilde{d}\right\}+p^{2}\left\{-k_{s 1}+\psi_{1} k_{1}-\psi_{2}\right\}+k_{1} p e \psi_{2} \\
& -\psi_{1} k_{1}^{2} e(p-\dot{e}) \\
= & p\left\{\bar{u}_{s 2}-\varphi_{d}^{T} \widetilde{\psi}+\widetilde{d}\right\}+p^{2}\left\{-k_{s 1}+\psi_{1} k_{1}-\psi_{2}\right\}+k_{1} p e \psi_{2} \\
& -\psi_{1} k_{1}^{3} e^{2} .
\end{aligned}
$$

If $A_{1}$ in (26) is positive definite, then

$$
\dot{V}_{s} \leq p\left\{\bar{u}_{s 2}-\varphi_{d}^{T} \widetilde{\psi}+\widetilde{d}\right\}-k_{2} p^{2}-\frac{1}{2} \psi_{1} k_{1}^{3} e^{2} .
$$

With Condition (i) of (28) and $\lambda=\min \left\{2 k_{2} / \psi_{1 \max }, k_{1}\right\}$, the derivative of $V_{s}$ becomes

$$
\dot{V}_{s} \leq-\lambda V_{s}+\varepsilon,
$$

which leads to (32). Thus $p$ and $e$ are bounded. Since $x_{1 d}(t)$ is assumed to be a bounded signal with bounded derivatives up to the second order, noting (21), it follows that $y_{\mathrm{eq}}$ is bounded. Since $e=x_{1}-x_{1 d}$ and $p=x_{2}-y_{\mathrm{eq}}$, we can know that the state $x$ is bounded. From Property (P3) of (19), the boundedness of $\widehat{\psi}$ is apparent. The control input $\bar{u}$ is thus bounded. This proves (A) of Theorem 3. Now consider the situation in (B) 
of Theorem 3 , that is, $\widetilde{d}=0, \forall t \geq t_{0}$, then choose a positive definite function $V_{a}$ as follows:

$$
V_{a}=V_{s}+\frac{1}{2} \widetilde{\psi}^{T} \Gamma^{-1} \widetilde{\psi}
$$

From (35), Condition (ii) of (28), and Property (P3) of (19), the derivative of $V_{a}$ satisfies

$$
\dot{V}_{a} \leq-k_{2} p^{2}-\frac{1}{2} \psi_{1} k_{1}^{2} e^{2}+\widetilde{\psi}^{T} \Gamma^{-1}(\dot{\hat{\psi}}-\Gamma \tau) \leq-W
$$

where $W=k_{2} p^{2}+\psi_{1} k_{1}^{3} e^{2} / 2$. Therefore, $W \in L_{2}$ and $V_{a} \in L_{\infty}$. Since all signals are bounded, it is easy to check that $\dot{W}$ is bounded and thus uniformly continuous. By applying Barbalat's lemma [31], $W \rightarrow 0$ as $t \rightarrow \infty$, which implies that the conclusion of (B) of Theorem 3 is true.

Remark 4. Results in (A) of Theorem 3 indicate that the proposed controller has an exponentially converging transient performance with the exponentially converging rate $\lambda$ and the final tracking error is able to be adjusted via certain controller parameters freely in a known form; it is seen from (32) that $\lambda$ can be made arbitrarily large, and $\varepsilon / \lambda$, the bound of $V(\infty)$ (an index for the final tracking errors), can be made arbitrarily small by increasing feedback gains $k_{2}$ and/or decreasing controller parameter $\varepsilon$. Such a guaranteed transient performance is especially important for the control of electrohydraulic systems since execute time of a run is very short. (B) of Theorem 3 implies that the parametric uncertainties may be reduced through parameter adaptation and an improved performance is obtained.

\section{Simulation Results}

To illustrate above designs, simulation results are obtained for the hydraulic system discussed in Section 2, which has the following actual parameters: $J=30 \mathrm{~kg} \cdot \mathrm{m}^{2}, D_{m}=1.9167 \times$ $10^{-4} \mathrm{~m}^{3} / \mathrm{rad}, B_{m}=45 \mathrm{~N} \cdot \mathrm{m} \cdot \mathrm{s} / \mathrm{rad}, 4 \beta_{e} / V_{m}=4.05 \times 10^{12} \mathrm{~N} / \mathrm{m}^{5}$, $C_{\mathrm{sl}}=9.5 \times 10^{-11} \mathrm{~m}^{5} /(\mathrm{N} \cdot \mathrm{s}), k_{\mathrm{to}}=3.765 \times 10^{-8} \mathrm{~m}^{4} /(\mathrm{s} \cdot \mathrm{V} \cdot \sqrt{\mathrm{N}})$, $P_{s}=21 \times 10^{6} \mathrm{~Pa}$, and $\tau_{v}=0.005 \mathrm{~s}$. The bounds of uncertain ranges are given by $J_{m \max }=40, J_{m \min }=20, B_{m \max }=60$, $B_{m \text { min }}=30, T_{\text {ed } \max }=1000, T_{\text {ed min }}=-1000$, and $\delta_{d}=2$. The initial estimates of uncertain parameters are chosen as $J_{m \text { ini }}=$ $20, B_{m \text { ini }}=30$, and $T_{\text {ed ini }}=0$, which satisfy (15) but differ significantly from their actual values to test the effect of parametric uncertainties. A sampling period of $0.2 \mathrm{~ms}$ is used in all simulation.

In general, hydraulic motion servo system usually suffers from significant external disturbance, which could degrade the performance of hydraulic motion servo system. As an example of varying boundary discontinuous projection, ignoring the minor factor of unmodeled friction torques, that is, assume $\tilde{f}(t, \theta, \dot{\theta})=0$, the varying boundary of external disturbance $T_{\text {ed }}$ can be estimated online by

$$
\begin{aligned}
& T_{\text {ed_max }}=\alpha_{\text {up }}\left(J_{m} \ddot{\theta}+B_{m} \dot{\theta}-P_{L} D_{m}\right), \quad \alpha_{\text {up }}>1, \\
& T_{\text {ed_min }}=\alpha_{\text {down }}\left(J_{m} \ddot{\theta}+B_{m} \dot{\theta}-P_{L} D_{m}\right), \quad 0<\alpha_{\text {down }}<1,
\end{aligned}
$$

where $\alpha_{\text {up }}$ and $\alpha_{\text {down }}$ are, respectively, scaled factors of estimated external disturbance. And $\alpha_{\text {up }}=1.1$ and $\alpha_{\text {down }}=0.9$ are used in the simulation. Meanwhile, noting that $k_{u}$ is a varying parameter, so it is necessary to calculate $k_{u}$ online via (11) by employing the load pressure feedback.

In implementation, as an alternative, a pragmatic approach $[1,2]$ is utilized to simply choose $k_{s 1}$ large enough combined with $k_{s 2}$ without worrying about the conditions (26), (28), and (29). And the trajectory initialization has been applied for satisfying condition (23), to further reduce the transient tracking error. And the trajectory generator in [2] is used in this paper to complete this mission.

The following three controllers are compared.

(1) $\operatorname{ARC}(v)$ : the simplified DCARC with varying boundary discontinuous projection, which is proposed in this paper. The controller parameters are: $k_{1}=30$, $k_{s 1}=400$, and $\Gamma=\operatorname{diag}\left\{1 \times 10^{5}, 1 \times 10^{5}, 1 \times 10^{8}\right\}$.

(2) DCARC: the desired compensation adaptive robust control with traditional discontinuous projection and the controller parameters are the same as in $\operatorname{ARC}(v)$.

(3) Motion controller: the proportional-integral-derivative (PID) controller, which is widely used in industrial control. The tuned gains are $k_{P}=100, k_{I}=10$, and $k_{D}=1$, which represent the $P$-gain, $I$-gain, and $D$-gain, respectively.

To test the normal tracking performance of each controller, simulations are first run for the ideal case only with parametric uncertainties (i.e., $T_{\text {ed }}=0$ ). The desired trajectory is a sinusoidal curve given by $x_{1 d}=0.5 \sin (t)$. The tracking errors are shown in Figure 2. As shown, all ARC controllers have very small tracking errors, in contrast to the large tracking errors achieved by traditional PID controller, and this verifies the excellent tracking capability of the proposed algorithms and the effectiveness of introducing parameter adaptation. Furthermore, except the very short beginning period, ARC $(v)$ has the same performance with DCARC.

To test the robustness against performance of the proposed schemes, a large varying external disturbance sinusoidal $T_{\text {ed }}$ with amplitude of $800 \mathrm{Nm}$ and frequency of $31.4 \mathrm{rad} / \mathrm{s}$ is added to the system. As shown in Figure 3, all ARC controllers still have very small tracking errors in spite of the added large disturbance. Furthermore, comparing $\operatorname{ARC}(v)$ with DCARC, it is seen that $\operatorname{ARC}(v)$ has a much shorter recovery period and a smaller transient tracking error, due to the different qualitative parameter adaptation transient of the two schemes when the system is subjected to large disturbance. As shown in Figure 4, the proposed ARC $(v)$ scheme guarantees that the parameter estimates stay within the varying boundary range all the time, while the parameter estimates in DCARC with fixed boundary range result in a terrible parameter adaptation. This verifies that the varying boundary discontinuous projection based ARC has a more robust parameter adaptation process. Consequently, a better performance can be expected.

The simulation is also run for fast desired trajectory and similar results have been obtained. For example, for a $2 \mathrm{~Hz}$ desired trajectory given by $x_{1 d}=0.2 \sin (4 \pi t)$, the tracking 

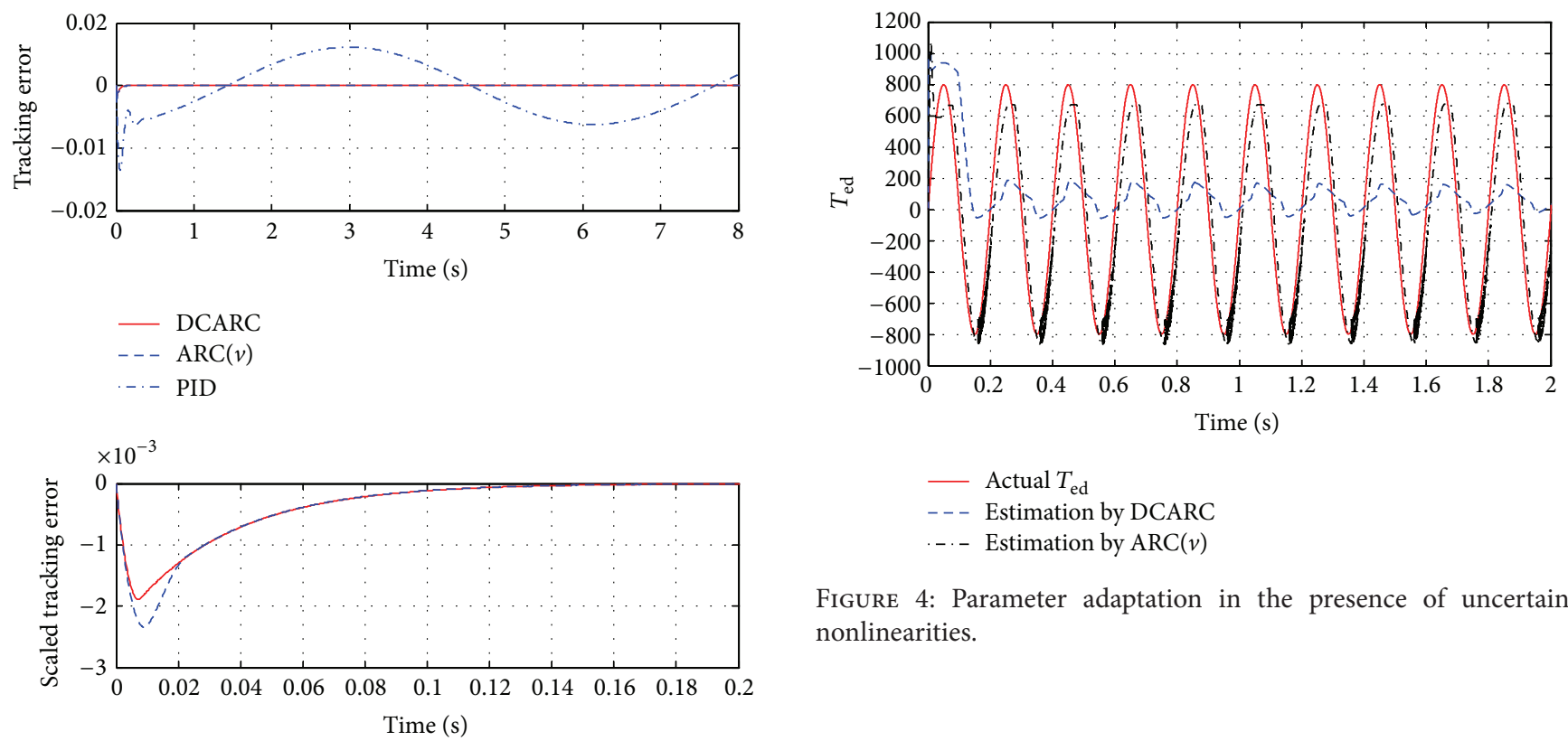

- Actual $T_{\text {ed }}$

- - Estimation by DCARC

...- Estimation by $\operatorname{ARC}(v)$

FIgURE 4: Parameter adaptation in the presence of uncertain nonlinearities.

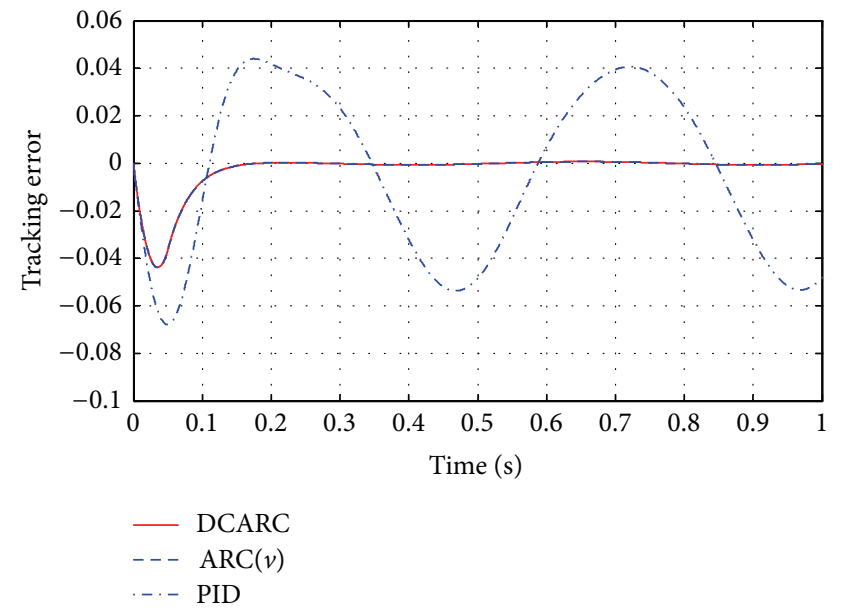

FIGURE 5: Tracking errors for a fast sinusoidal curve in the presence of parametric uncertainties only.

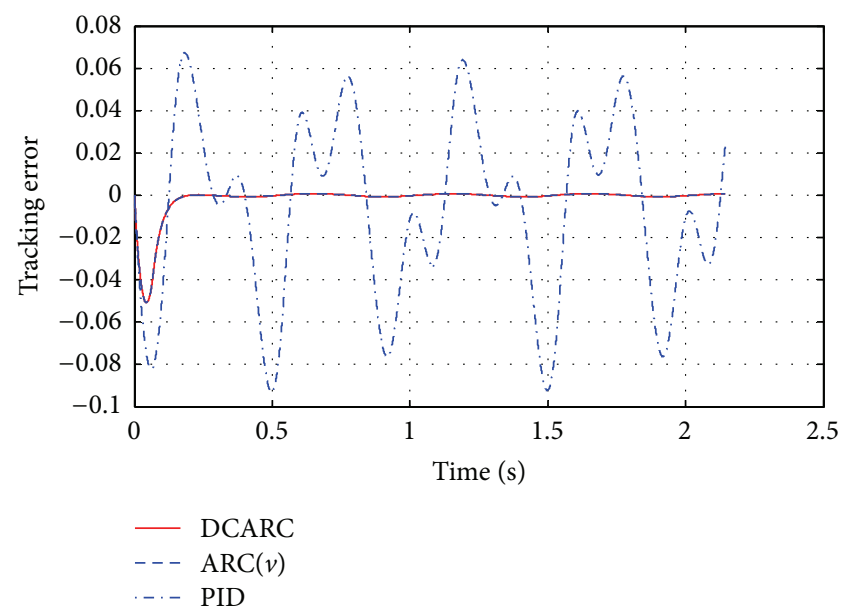

FIGURE 6: Tracking errors for a fast sinusoidal curve in the presence of parametric uncertainties and uncertain nonlinearities. 


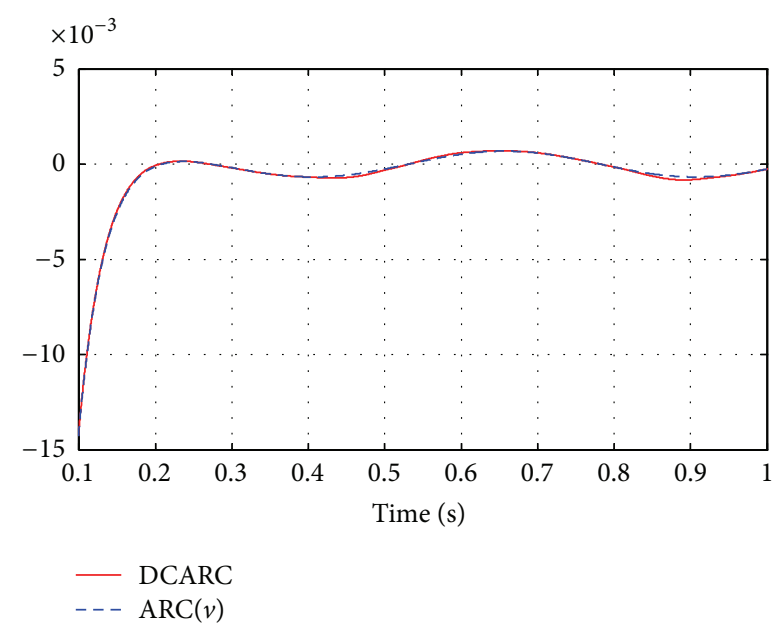

FIGURE 7: Scaled ARCs' tracking errors for a fast sinusoidal curve in the presence of parametric uncertainties and uncertain nonlinearities.
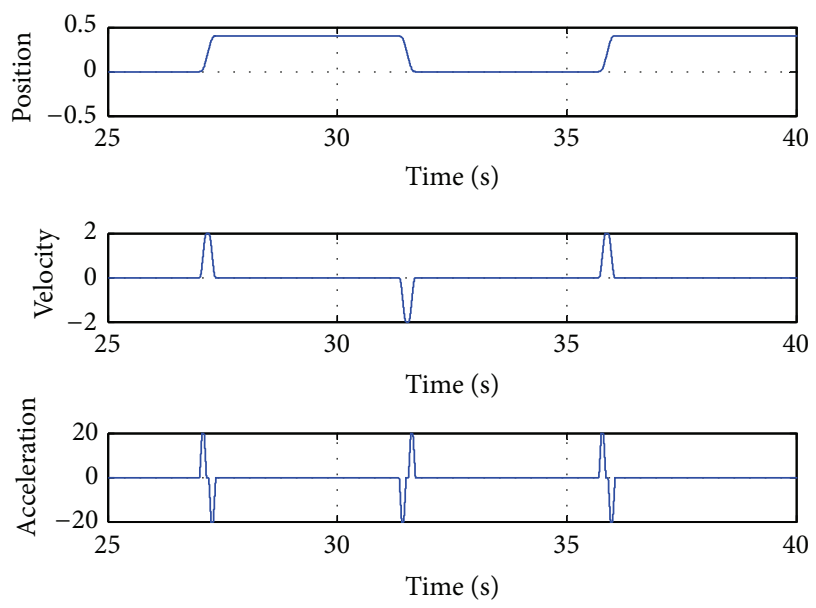

FIGURE 8: Point to point motion trajectory profile.

in Figure 8 , which has a maximum speed of $2 \mathrm{rad} / \mathrm{s}$ and a maximum acceleration of $20 \mathrm{rad} / \mathrm{s}^{2}$. The tracking errors are shown in Figures 9, 10, 11, and 12 with or without external disturbance. As seen, during the start and the end period when the system experiences large acceleration and deceleration, transient tracking errors become a little larger. Again, ARC $(v)$ has a better tracking performance than DCARC when system suffers large external disturbance.

\section{Conclusions}

In this paper, a varying boundary discontinuous projection based adaptive robust controller is proposed for the high performance robust motion control of an electrohydraulic servo system driven by dual vane hydraulic rotary actuator, to enhance the robustness against parameter variations and disturbances. And some significant modifications and simplifications have been made by using differential pressure signal according to the internal leakage feature of the considered

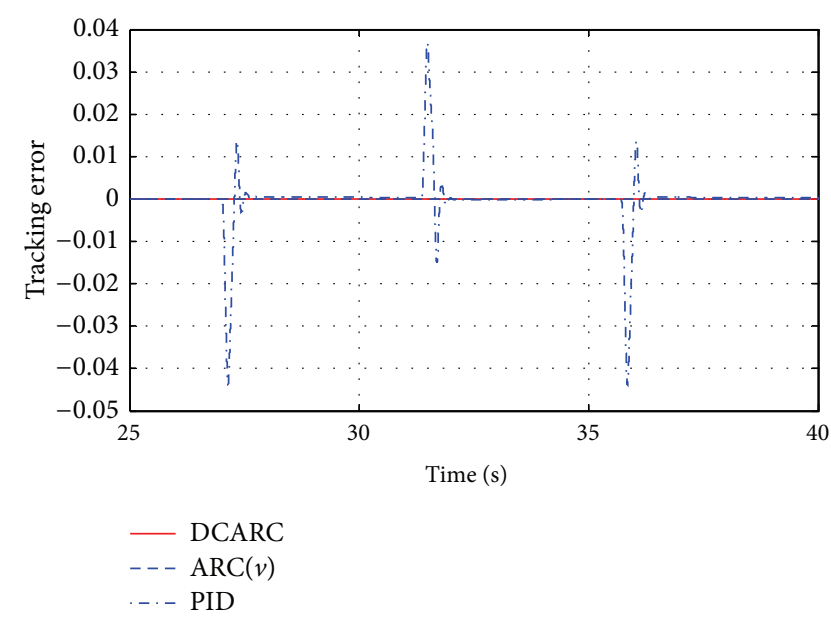

FIGURE 9: Tracking errors in point to point motion in the presence of parametric uncertainty only.

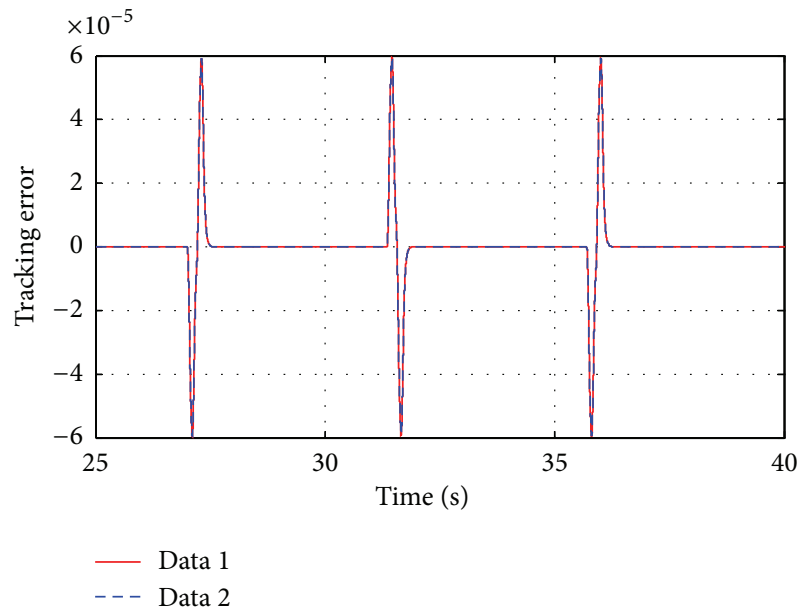

FIGURE 10: Scaled ARCs' tracking errors in point to point motion in the presence of parametric uncertainty only.

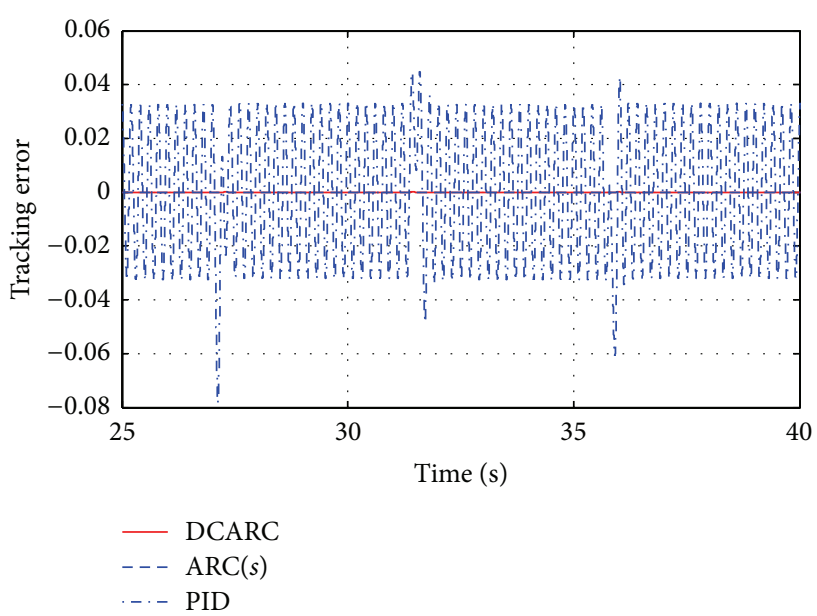

FIGURE 11: Tracking errors in point to point motion in the presence of parametric uncertainty and uncertain nonlinearities. 


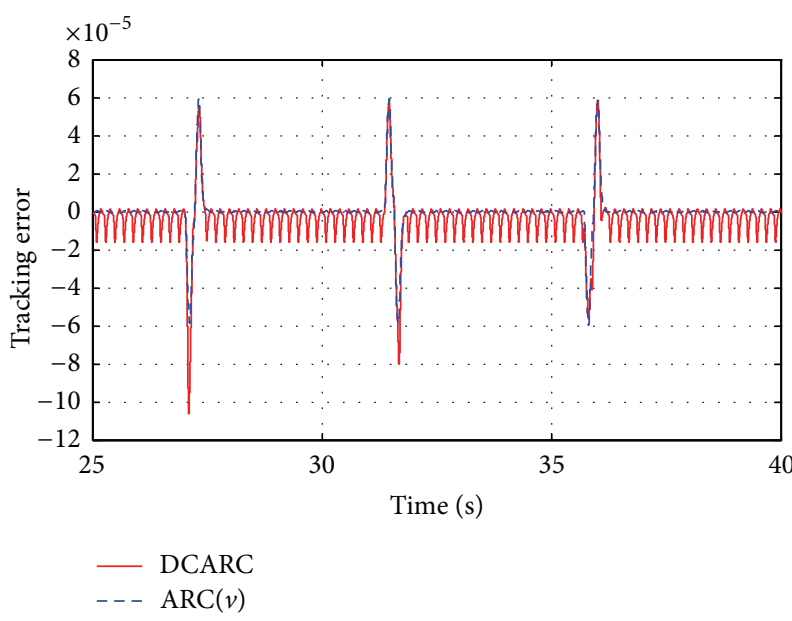

FIGURE 12: Scaled ARCs' tracking errors in point to point motion in the presence of parametric uncertainty and uncertain nonlinearities.

hydraulic rotary actuator. Based on a simplified system model and varying boundary discontinuous projection, a desired compensation ARC controller is constructed. The controller takes into account the particular nonlinearities associated with hydraulic dynamics and parametric uncertainties due to variations of inertia load and lumped damping coefficient as well as uncertain nonlinearities coming from external disturbance. Comparative simulation results show that the proposed scheme has a more robust parameter adaptation process and is more suitable for implementation. As future works, it is very interesting to investigate the adaptive and robust design of hydraulic systems with potential hydraulic faults [41-44] to improve the safety for critical-safe systems.

\section{Conflict of Interests}

The authors declare that there is no conflict of interests regarding the publication of this paper.

\section{References}

[1] J. Yao, Z. Jiao, D. Ma, and L. Yan, "High-accuracy tracking control of hydraulic rotary actuators with modelling uncertainties," IEEE/ASME Transactions on Mechatronics, vol. 19, no. 2, pp. 633-641, 2014.

[2] B. Yao, F. Bu, J. Reedy, and G. T.-. Chiu, "Adaptive robust motion control of single-rod hydraulic actuators: theory and experiments," IEEE/ASME Transactions on Mechatronics, vol. 5, no. 1, pp. 79-91, 2000.

[3] J. E. Bobrow and K. Lum, "Adaptive, high bandwidth control of a hydraulic actuator," ASME Journal of Dynamic Systems, Measurement and Control, vol. 118, no. 4, pp. 714-720, 1996.

[4] J. Yao, Z. Jiao, and D. Ma, "Extended-state-observer-based output feedback nonlinear robust control of hydraulic systems with backstepping," IEEE Transactions on Industrial Electronics, vol. 61, no. 11, pp. 6285-6293, 2014.

[5] W. Sun, H. Gao Sr., and O. Kaynak, "Finite frequency $H_{\infty}$ control for vehicle active suspension systems," IEEE Transactions on Control Systems Technology, vol. 19, no. 2, pp. 416-422, 2011.
[6] A. Alleyne and J. K. Hedrick, "Nonlinear adaptive control of active suspensions," IEEE Transactions on Control Systems Technology, vol. 3, no. 1, pp. 94-101, 1995.

[7] W. Sun, Y. Zhao, J. Li, L. Zhang, and H. Gao, "Active suspension control with frequency band constraints and actuator input delay," IEEE Transactions on Industrial Electronics, vol. 59, no. 1, pp. 530-537, 2012.

[8] J. Yao, Z. Jiao, and S. Han, "Friction compensation for low velocity control of hydraulic flight motion simulator: a simple adaptive robust approach," Chinese Journal of Aeronautics, vol. 26, no. 3, pp. 814-822, 2013.

[9] A. Alleyne, "Nonlinear force control of an electro-hydraulic actuator," in Proceedings of the Japan-USA Symposium on Flexible Automation, pp. 193-200, Boston, Mass, USA, July 1996.

[10] A. R. Plummer, "Robust electrohydraulic force control," Proceedings of the Institution of Mechanical Engineers part I: Journal of Systems and Control Engineering, vol. 221, no. 4, pp. 717-731, 2007.

[11] J. Yao, Z. Jiao, Y. Shang, and C. Huang, "Adaptive nonlinear optimal compensation control for electro-hydraulic load simulator," Chinese Journal of Aeronautics, vol. 23, no. 6, pp. 720-733, 2010.

[12] N. Niksefat and N. Sepehri, "Design and experimental evaluation of a robust force controller for an electro-hydraulic actuator via quantitative feedback theory," Control Engineering Practice, vol. 8, no. 12, pp. 1335-1345, 2000.

[13] Y. Nam and S. K. Hong, "Force control system design for aerodynamic load simulator," Control Engineering Practice, vol. 10, no. 5, pp. 549-558, 2002.

[14] K. K. Ahn, D. Q. Truong, T. Q. Thanh, and B. R. Lee, "Online self-tuning fuzzy proportional-integral-derivative control for hydraulic load simulator," Proceedings of the Institution of Mechanical Engineers I: Journal of Systems and Control Engineering, vol. 222, no. 2, pp. 81-95, 2008.

[15] J. Yao, Z. Jiao, and D. Ma, "High dynamic adaptive robust control of load emulator with output feedback signal," Journal of the Franklin Institute. Engineering and Applied Mathematics, vol. 351, no. 8, pp. 4415-4433, 2014.

[16] W. Kim, D. Won, and C. C. Chung, "High gain observer based nonlinear position control for electro-hydraulic servo systems," in Proceedings of the American Control Conference (ACC'10), pp. 1440-1446, Baltimore, Md, USA, July 2010.

[17] J. Heintze, G. van Schothorst, A. J. J. v.d. Weiden, and P. C. Teerhuis, "Modeling and control of an industrial hydraulic rotary vane actuator," in Proceedings of the 32nd IEEE Conference on Decision and Control, pp. 1913-1918, December 1993.

[18] J. Yao, Z. Jiao, and B. Yao, "Robust control for static loading of electro-hydraulic load simulator with friction compensation," Chinese Journal of Aeronautics, vol. 25, no. 6, pp. 954-962, 2012.

[19] H. E. Merritt, Hydraulic Control Systems, Wiley, New York, NY, USA, 1967.

[20] W. Sun, H. Gao, and O. Kaynak, "Adaptive backstepping control for active suspension systems with hard constraints," IEEE/ASME Transactions on Mechatronics, vol. 18, no. 3, pp. 1072-1079, 2013.

[21] J. Yao, Z. Jiao, and B. Yao, "Nonlinear adaptive robust backstepping force control of hydraulic load simulator: theory and experiments," Journal of Mechanical Science and Technology, vol. 28, no. 4, pp. 1499-1507, 2014.

[22] R. Vossoughi and M. Donath, "Dynamic feedback linearization for electro-hydraulically actuated control systems," ASME Journal of Dynamic Systems, Measurement, and Control, vol. 117, no. 4, pp. 468-477, 1995. 
[23] K. Ziaei and N. Sepehri, "Design of a nonlinear adaptive controller for an electrohydraulic actuator," Journal of Dynamic Systems, Measurement and Control, Transactions of the ASME, vol. 123, no. 3, pp. 449-456, 2001.

[24] J. Yao, Z. Jiao, and D. Ma, "RISE-based precision motion control of DC motors with continuous friction compensation," IEEE Transactions on Industrial Electronics, 2014.

[25] W. Sun, H. Gao, and B. Yao, "Adaptive robust vibration control of full-car active suspensions with electro-hydraulic actuators," IEEE Transactions on Control Systems Technology, vol. 21, no. 6, pp. 2417-2422, 2013.

[26] B. Yao and M. Tomizuka, "Adaptive robust control of SISO nonlinear systems in a semi-strict feedback form," Automatica, vol. 33, no. 5, pp. 893-900, 1997.

[27] B. Yao, "Desired compensation adaptive robust control," Journal of Dynamic Systems, Measurement and Control, Transactions of the ASME, vol. 131, no. 6, Article ID 061001, 7 pages, 2009.

[28] B. Yao and M. Tomizuka, "Smooth robust adaptive sliding mode control of manipulators with guaranteed transient performance," Journal of Dynamic Systems, Measurement and Control, Transactions of the ASME, vol. 118, no. 4, pp. 764-775, 1996.

[29] B. Yao and C. Jiang, "Advanced motion control: from classical PID to nonlinear adaptive robust control," in Proceedings of the 2010 11th IEEE International Workshop on Advanced Motion Control (AMC '10), pp. 815-829, Nagaoka, Japan, March 2010.

[30] J. J. E. Slotine and W. Li, "Adaptive manipulator control: a case study," IEEE Transactions on Automatic Control, vol. 33, no. 11, pp. 995-1003, 1988.

[31] M. Krstic, I. Kanellakopoulos, and P. V. Kokotovic, Nonlinear and Adaptive Control Design, Wiley, New York, NY, USA, 1995.

[32] M. J. Corless and G. Leitmann, "Continuous state feedback guaranteeing uniform ultimate boundedness for uncertain dynamic systems," IEEE Transactions on Automatic Control, vol. 26, no. 5, pp. 1139-1144, 1981.

[33] J. Yao, Z. Jiao, and D. Ma, "Adaptive robust control of DC motors with extended state observer," IEEE Transactions on Industrial Electronics, vol. 61, no. 7, pp. 3630-3637, 2014.

[34] W. Sun, Z. Zhao, and H. Gao, "Saturated adaptive robust control for active suspension systems," IEEE Transactions on Industrial Electronics, vol. 60, no. 9, pp. 3889-3896, 2013.

[35] L. Lu, Z. Chen, B. Yao, and Q. Wang, "Desired compensation adaptive robust control of a linear-motor-driven precision industrial gantry with improved cogging force compensation," IEEE/ASME Transactions on Mechatronics, vol. 13, no. 6, pp. 617624, 2008.

[36] J. Yao, G. Yang, Z. Jiao, and D. Ma, "Adaptive robust motion control of direct-drive DC motors with continuous friction compensation," Abstract and Applied Analysis, vol. 2013, Article ID 837548, 14 pages, 2013.

[37] A. Mohanty and B. Yao, "Indirect adaptive robust control of hydraulic manipulators with accurate parameter estimates," IEEE Transactions on Control Systems Technology, vol. 19, no. 3, pp. 567-575, 2011.

[38] I. Uzmay and R. Burkan, "Parameter estimation and upper bounding adaptation in adaptive-robust control approaches for trajectory control of robots," Robotica, vol. 20, no. 6, pp. 653660, 2002.

[39] X. Zhu, G. Tao, B. Yao, and J. Cao, "Integrated direct/indirect adaptive robust posture trajectory tracking control of a parallel manipulator driven by pneumatic muscles," IEEE Transactions on Control Systems Technology, vol. 17, no. 3, pp. 576-588, 2009.
[40] J. Yao, Z. Jiao, B. Yao, Y. Shang, and W. Dong, "Nonlinear adaptive robust force control of hydraulic load simulator," Chinese Journal of Aeronautics, vol. 25, no. 5, pp. 766-775, 2012.

[41] A. Y. Goharrizi and N. Sepehri, "Internal leakage detection in hydraulic actuators using empirical mode decomposition and hilbert spectrum," IEEE Transactions on Instrumentation and Measurement, vol. 61, no. 2, pp. 368-378, 2012.

[42] P. Goupil, "Oscillatory failure case detection in the A380 electrical flight control system by analytical redundancy," Control Engineering Practice, vol. 18, no. 9, pp. 1110-1119, 2010.

[43] L. Lavigne, A. Zolghadri, P. Goupil, and P. Simon, "A modelbased technique for early and robust detection of oscillatory failure case in A380 actuators," International Journal of Control, Automation and Systems, vol. 9, no. 1, pp. 42-49, 2011.

[44] J. Yao, G. Yang, and D. Ma, "Internal leakage fault detection and tolerant control of single-rod hydraulic actuators," Mathematical Problems in Engineering, vol. 2014, Article ID 345345, 14 pages, 2014. 


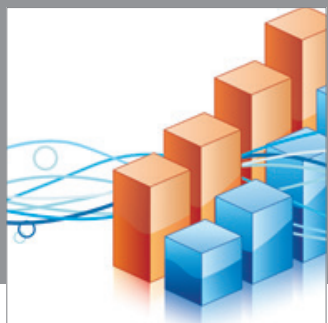

Advances in

Operations Research

mansans

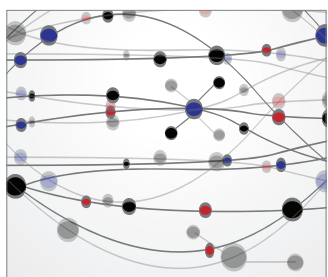

The Scientific World Journal
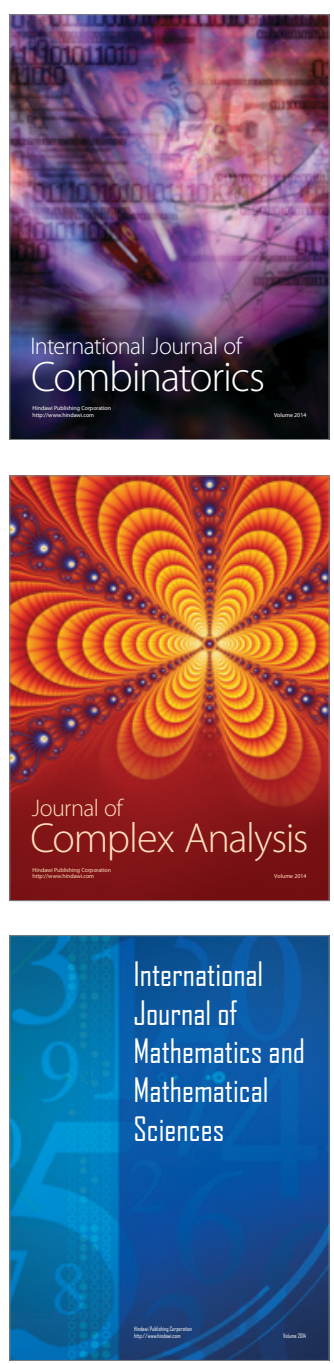
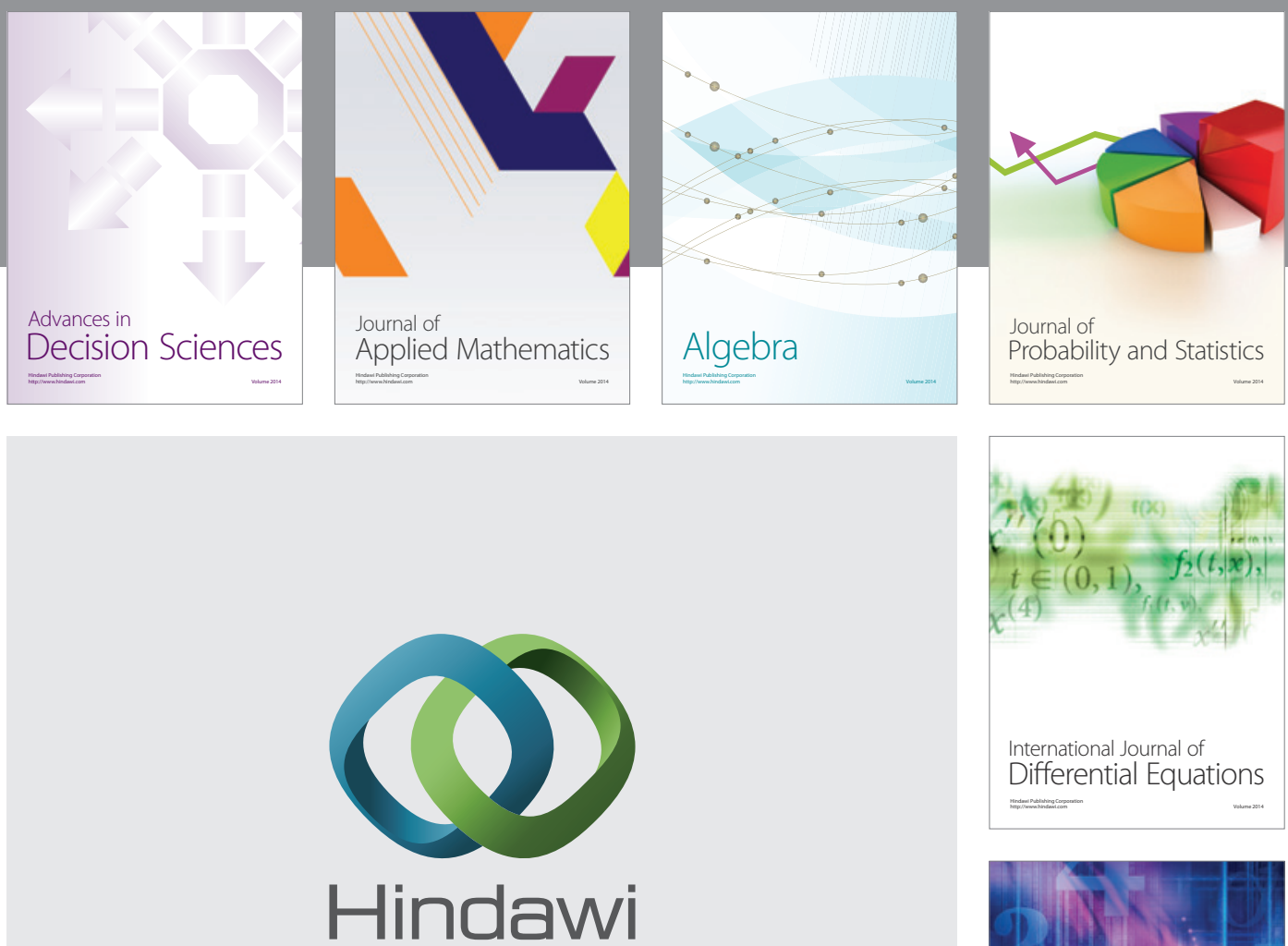

Submit your manuscripts at http://www.hindawi.com
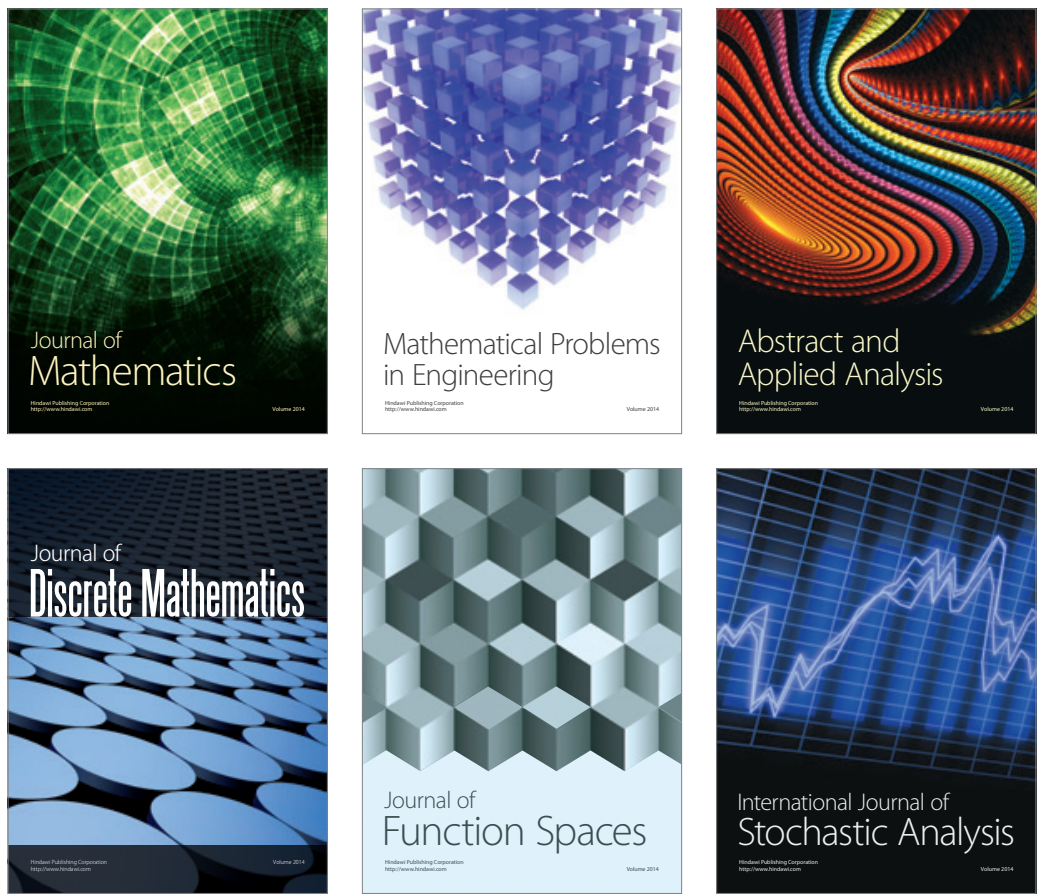

Journal of

Function Spaces

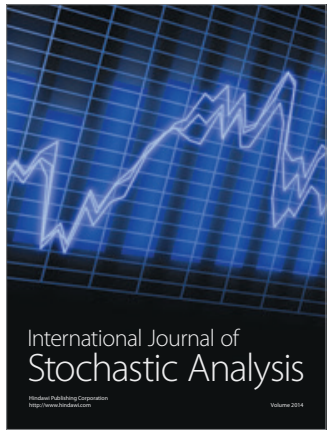

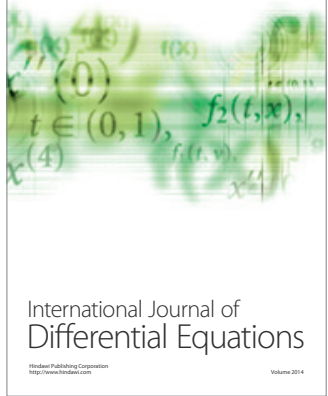
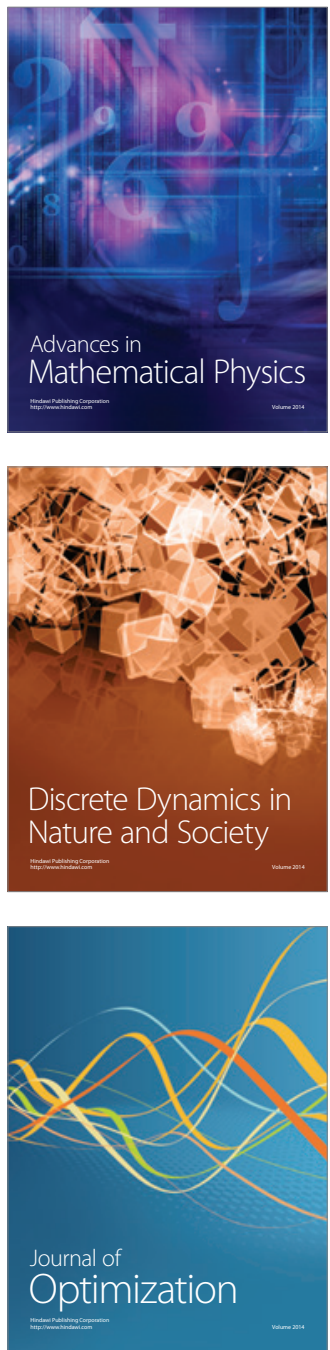